\title{
Incision ante- et post-capture dans les vallées de l'Ornain \\ et de la Saulx (Est du bassin de Paris)
}

Ante- and post-capture incision of the Ornain and Saulx valleys (East of the Paris basin)

Einschneidung vor und nach der Anzapfung in den Tälern des Ornain und der Saulx (Osten des Pariser Beckens)

Dominique Harmand, Pierre-Jean Fauvel, Stéphane Jaillet, Jacques Le Roux, Jacques Allouc, Jacques Brulhet et Marcel Brocandel

\section{OpenEdition \\ Journals}

Édition électronique

URL : http://journals.openedition.org/rge/2669

DOI : $10.4000 /$ rge.2669

ISSN : $2108-6478$

Éditeur

Association des géographes de l'Est

Édition imprimée

Date de publication : 1 septembre 2002

ISSN : 0035-3213

\section{Référence électronique}

Dominique Harmand, Pierre-Jean Fauvel, Stéphane Jaillet, Jacques Le Roux, Jacques Allouc, Jacques Brulhet et Marcel Brocandel, «Incision ante- et post-capture dans les vallées de l'Ornain

et de la Saulx (Est du bassin de Paris) », Revue Géographique de l'Est [En ligne], vol. 42 / 4 | 2002, mis en ligne le 17 janvier 2011, consulté le 08 septembre 2020. URL : http://journals.openedition.org/rge/ 2669 ; DOI : https://doi.org/10.4000/rge.2669

Ce document a été généré automatiquement le 8 septembre 2020

Tous droits réservés 


\section{Incision ante- et post-capture dans les vallées de l'Ornain et de la Saulx (Est du bassin de Paris)}

Ante- and post-capture incision of the Ornain and Saulx valleys (East of the

Paris basin)

Einschneidung vor und nach der Anzapfung in den Tälern des Ornain und der

Saulx (Osten des Pariser Beckens)

Dominique Harmand, Pierre-Jean Fauvel, Stéphane Jaillet, Jacques Le Roux, Jacques Allouc, Jacques Brulhet et Marcel Brocandel

\section{NOTE DE L'AUTEUR}

Cette recherche effectuée dans les vallées de l'Ornain en aval de Gondrecourt-leChâteau, de la Saulx en aval de Stainville, et de l'Ornain-Saulx, au Nord de Revigny-surOrnain, s'inscrit dans le programme des études entreprises par l'ANDRA (Agence Nationale pour la gestion des Déchets RAdioactifs) autour du laboratoire souterrain de Bure (Meuse) situé dans le bassin de la Saulx. Les recherches sur le concept de stockage en formation géologique profonde nécessitent de tenir compte de l'évolution naturelle du site dans le futur. La présente étude participe aux travaux entrepris par un groupement de laboratoires pour préciser la dynamique de l'évolution géomorphologique au cours du passé, en vue de son extrapolation vers le futur, notamment en fonction de l'évolution climatique. Le volet présenté ici, qui porte sur les stades d'incision des vallées de l'Ornain et de la Saulx, associe des laboratoires des Universités de Nancy 1, de Nancy 2 et de l'Institut Polytechnique de Lorraine (Ecole Nationale Supérieure de Géologie). 


\section{Présentation et objectifs}

1 Les vallées de l'Ornain et de la Saulx se situent dans l'Est du bassin de Paris, aux confins $\mathrm{du}$ Barrois (Lorraine méridionale), où affleurent des séries marno-calcaires du Jurassique, et du Perthois (Champagne humide), où prédominent les séries argilosableuses du Crétacé inférieur. Dans la région de Revigny-sur-Ornain, les deux cours d'eau sont marqués par l'existence d'un coude de capture attestant leur prolongement ancien vers l'Aisne (fig. 1).

Figure 1 : Cadre géographique de l'étude

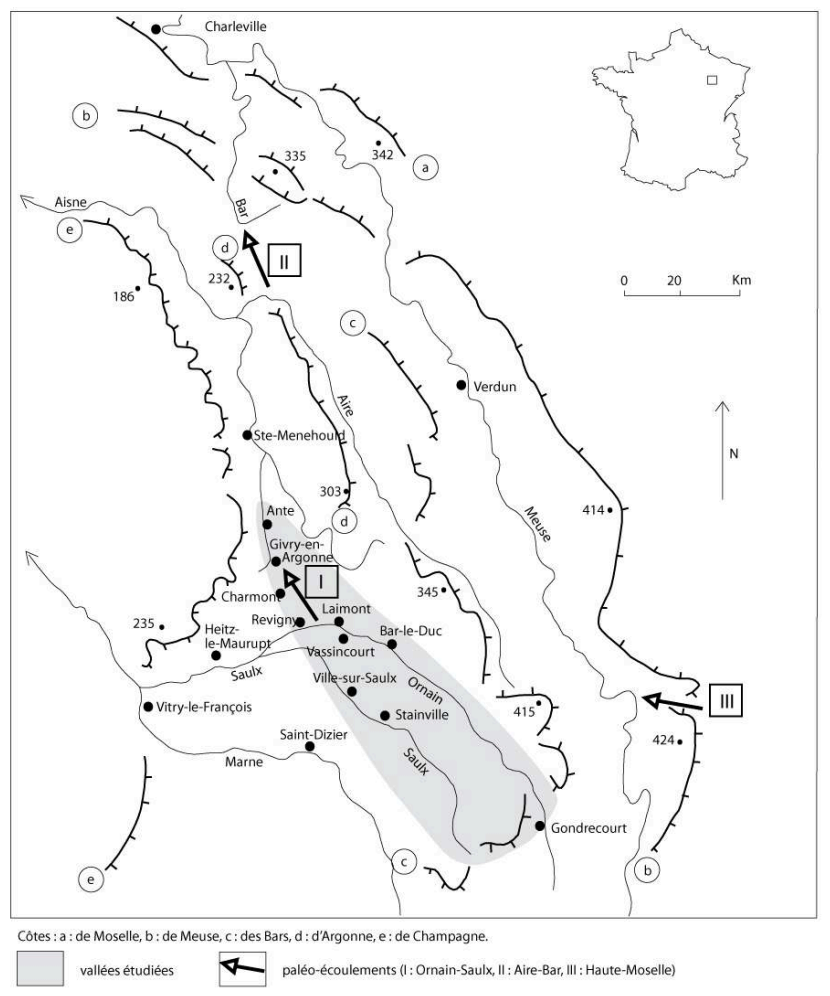

2 A partir des alignements hydrographiques Saulx et Ornain supérieurs - Ante Aisne inférieure, et des vastes placages alluviaux situés entre l'Ornain et l'Aisne, maints auteurs (Buvignier, 1852 ; Denizot, 1919 ; Gardet, 1937 ; Pâque et Cailleux, 1946 ; Tricart, 1948 ; Blondeau et al., 1988 ; Laurain et al., 1998) mettent en évidence un paléo-écoulement de la Saulx et de l'Ornain supérieurs vers l'Aisne.

3 La capture de la Saulx et de l'Ornain a été expliquée par les difficultés de creusement de l'Aisne dans la gaize de l'Argonne, ce qui aurait entravé en amont l'incision de la Saulx et de l'Ornain (Tricart, 1948). A l'opposé, la Vière, qui a réalisé la capture de la Saulx et de l'Ornain au profit de la Marne, a bénéficié de la présence, dans la Champagne humide, d'un substratum argilo-marneux facile à déblayer. D'après J. Tricart, la capture s'est effectuée par érosion régressive. Les découvertes paléontologiques et archéologiques effectuées dans les alluvions anté-capture (Gardet, 1937 ; Patte, 1937 ; Guillaume, 1982) permettent de rattacher celles-ci au Quaternaire moyen.

4 Cependant, les «traces morphologiques indiscutables des écoulements passés» (Weisrock, 1997) indiquent que la capture de la Saulx et de l'Ornain ne représente qu'une étape dans les réorganisations hydrographiques qui ont démantelé la partie 
occidentale du paléo-bassin de la Meuse (fig. 1). Les méandres surdimensionnés de la vallée de la Bar (Davis, 1895 ; Bois, 1903 ; Deshaies et Weisrock, 1995), de même que la diminution brusque de la tourmaline à $+30 \mathrm{~m}$ dans les alluvions des terrasses de la Meuse ardennaise, à proximité de la frontière franco-belge (Pissart et al., 1997) mettent en évidence un paléo-écoulement de l'Aisne supérieure, voire de la Marne supérieure, vers la Meuse au Quaternaire ancien. Après la capture de «l'Aisne-OrnainSaulx » (Tricart, 1948) au profit du bassin de la Seine, la vallée de « l'Aire-Bar » (Blache, 1943) a continué à être drainée vers la Meuse, jusqu'à ce que l'Aire fût capturée par l'Aisne.

5 Jusqu'à ce jour, les alluvions antérieures et postérieures à la capture de la Saulx et de l'Ornain n'avaient pas donné lieu à une cartographie systématique (Demassieux, 1969 ; Maubeuge, 1965, 1970 ; Stchépinsky, 1959, 1962) si bien qu'on ne disposait d'aucune datation relative des terrasses pour les vallées de la Saulx, de l'Ornain et de l'Ornain/Saulx, section de vallée située entre la confluence de ces deux cours d'eau et la vallée de l'Aisne.

6 Aussi, les objectifs de cette recherche ont-ils consisté à cartographier précisément les alluvions de ces trois sections de vallées (Harmand et Fauvel, 2001), notamment sur le territoire de la feuille de Revigny-sur-Ornain (Allouc et al., à paraitre), puis à reconstituer les stades de creusement des trois cours d'eau et à préciser les modalités de la capture de la Saulx et de l'Ornain, et enfin, à évaluer les taux d'incision anté- et post-capture dans le Barrois et le Perthois.

\section{Les terrasses alluviales des vallées de l'Ornain, de la Saulx et de l'Ornain/Saulx}

\section{A. Cartographie des alluvions anciennes des vallées de la Saulx, de I'Ornain et de l'Ornain/Saulx}

7 Dans les trois sections de vallées, la répartition des alluvions anciennes, constituées essentiellement de galets de calcaire tithonien, se calque sur les régions naturelles (fig. 1, 2). En amont, dans le Barrois, où les vallées de l'Ornain et de la Saulx sont fortement encaissées (environ $140 \mathrm{~m}$ pour l'Ornain au Nord-Ouest de Gondrecourt-le-Château, et $100 \mathrm{~m}$ pour la Saulx, en amont de Ville-sur-Saulx, au Sud-Ouest de Bar-le-Duc), les alluvions anciennes sont peu abondantes. En effet, sur les versants calcaires du Tithonien, les placages alluviaux sont discontinus et les alluvions ont une épaisseur faible, généralement métrique (Harmand et Fauvel, 2000, 2001). Elles sont toutefois plus abondantes sur les argiles et marnes du Kimméridgien de la vallée de l'Ornain. En aval, sur le site de capture de la Saulx et de l'Ornain, et au Sud-Ouest de l'Argonne, de vastes terrasses recouvrent les interfluves surbaissés modelés dans la gaize et dans les argiles albiennes. Les alluvions sont épaisses (10 m environ à Laimont, à Nettancourt, et à Givry-en-Argonne, d'après la Banque des Données du Sous-Sol du BRGM). Les alluvions grossières y sont surmontées par 1 à $2 \mathrm{~m}$ de limons. 
Figure 2 : Les alluvions anciennes des vallées de l'Ornain, de la Saulx, et de l'Ornain-Saulx

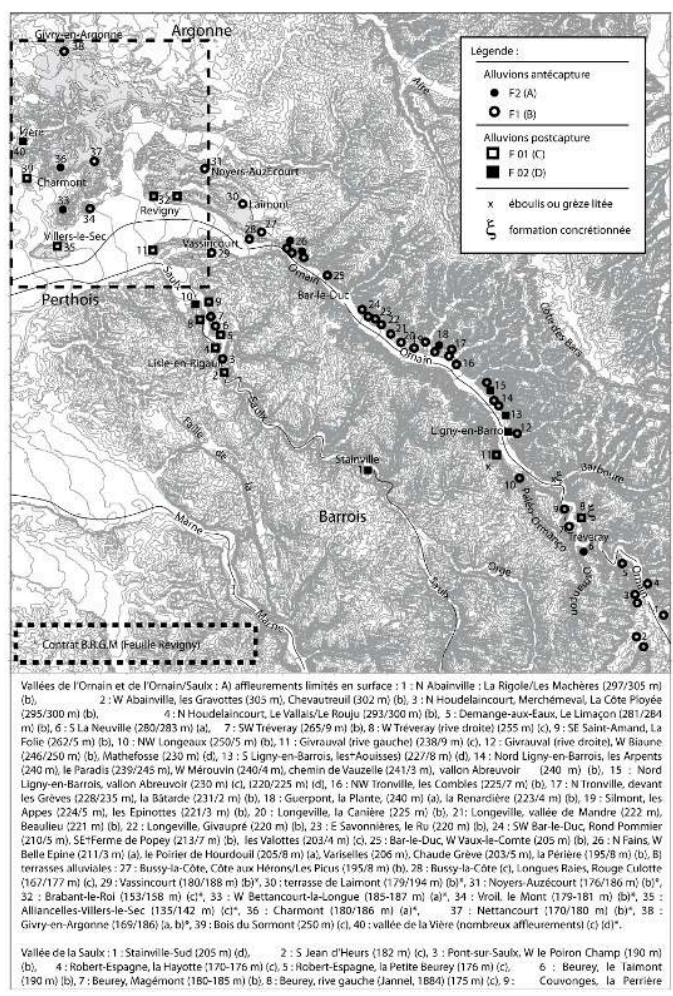

\section{B. Etagement des formations alluviales}

8 Dans la région du site de capture de la Saulx et de l'Ornain, les formations alluviales se répartissent en 7 formations alluviales anté-capture et post-capture constituées par 3 formations anté-capture étagées, notées $\mathrm{F} 3$ à $\mathrm{F} 1$ de la plus ancienne à la plus récente, et 4 formations post-capture, notées F 01 à F 04 (tableau 1). Ces 4 formations post-capture correspondent à 2 terrasses étagées et à 2 formations emboitées en fond de vallée (fig. 3, 4). Toutes ces formations correspondent à autant de stades d'incision des cours d'eau, notés respectivement de $\mathrm{Or} / \mathrm{Sa} 3$ (Or/Sa: Ornain/Saulx) à Or/Sa 1 pour les stades anté-capture et de Or 01 ou Sa 01 à Or 04 ou Sa 04 pour les stades post-capture. 
Tableau 1 : Les stades et épisodes d'incision des vallées de l'Ornain, de la Saulx et de l'Ornain/Saulx

\begin{tabular}{|c|c|c|c|c|c|}
\hline $\begin{array}{l}\text { Episodes } \\
\text { d'incision }\end{array}$ & $\begin{array}{c}\text { Formations } \\
\text { alluviales }\end{array}$ & $\begin{array}{c}\text { Stades d'incision } \\
\text { de l'Ornain/Saulx } \\
\text { (Paléo-Perthois et Perthois) }\end{array}$ & $\begin{array}{l}\text { Stades d'incision } \\
\text { vallée de la Saulx } \\
\text { (Stainville) }\end{array}$ & $\begin{array}{l}\text { Stades dincision } \\
\text { vallée de I'Ornain } \\
\text { (Tréveray) }\end{array}$ & Âge présumé \\
\hline D & & & & Or $7(+100 \mathrm{~m})^{*}$ & Pliocène? \\
\hline D & & & Sa $6(+75 \mathrm{~m})^{*}$ & Or $6(+80 \mathrm{~m}) *$ & Pliocène? \\
\hline C & & & Sa $5(+55 \mathrm{~m}) *$ & Or $5(+65 \mathrm{~m}) *$ & \\
\hline C & & & Sa $4(+45 \mathrm{~m}) *$ & Or $4(+45 \mathrm{~m}) *$ & \\
\hline C & F 3 & $\mathrm{Or} / \mathrm{Sa} 3(+20 \mathrm{~m})^{*}(+50 \mathrm{~m})^{* *}$ & $\mathrm{Sa} 3(+30 \mathrm{~m}) *$ & Or $3(+30 \mathrm{~m}) *$ & \\
\hline C & $\mathrm{F} 2$ & Or/Sa $2(+10 \mathrm{~m}) *(+50 \mathrm{~m}) * *$ & Sa $2(+15 \mathrm{~m}) *$ & Or $2(+15 \mathrm{~m})^{*}$ & \\
\hline C & $\mathrm{F} 1$ & $\mathrm{Or} / \mathrm{Sa} 1(+40 \mathrm{~m})^{* *}$ & Sa $1(+20 \mathrm{~m})^{* *}$ & Or $1(+10 \mathrm{~m})^{* *}$ & stades isotopiques $9 / 8$ \\
\hline \multicolumn{6}{|c|}{ Capture de la Saulx et de I'Ornain } \\
\hline B & $\mathrm{F} 01$ & Or $01(+15 / 20 m)^{* *}$ & Sa 01 & Or $01(+5 \mathrm{~m})^{* *}$ & stade isotopique $6 ?$ \\
\hline B & $\mathrm{F} 02$ & Or $02(+7 \mathrm{~m})^{* *}$ & $\mathrm{Sa} 02(+7 \mathrm{~m})^{* *}$ & Or 02 & stade isotopique $4 ?$ \\
\hline A & $\mathrm{F} 03$ & Or $03(+2 / 5 \mathrm{~m}) * *$ & $\mathrm{Sa} 03$ & Or 03 & Tardiglaciaire? \\
\hline A & $\mathrm{F} 04$ & Or 04 & Sa 04 & Or 04 & Holocène \\
\hline
\end{tabular}

* : altitude relative par rapport à la base des alluvions $\mathrm{F} 1$.

** : altitude relative par rapport au talweg.

9 Les formations anté-capture jalonnent un paléo-tracé de l'Ornain et de la Saulx vers le nord, les deux cours d'eau convergeant à Nettancourt où ils formaient l'OrnainSaulx. Ce dernier suivait, au Nord de Givry-en-Argonne, le tracé de la vallée actuelle de l'Ante et rejoignait l'Aisne à Sainte-Menehould, constituant avec celle-ci l'AisneOrnain-Saulx. Les alluvions post-capture suivent le tracé actuel de l'Ornain, de la Saulx, de la Chée et de la Vière.

Les formations anté-capture et post-capture ont le même lithofaciès et sont constituées surtout de galets calcaires issus du Tithonien, même dans la vallée de la Vière, où les dépôts post-capture sont formés d'alluvions de l'Ornain-Saulx remaniées. Toutefois, dans les sections de vallée étudiées, les formations alluviales les plus abondantes appartiennent à la formation $\mathrm{F} 1$, qui constitue la dernière nappe alluviale antecapture. La base de cette formation constitue donc un point de repère fondamental pour reconstituer le dispositif des terrasses de l'Ornain/Saulx. 
Figure 3 : Carte du site de capture de la Saulx et de l'Ornain : le Paléo-Perthois, des traces de 3 vallées anté-capture : Or/Sa 3, Or/Sa 2 et $\mathrm{Or} / \mathrm{Sa} 1$

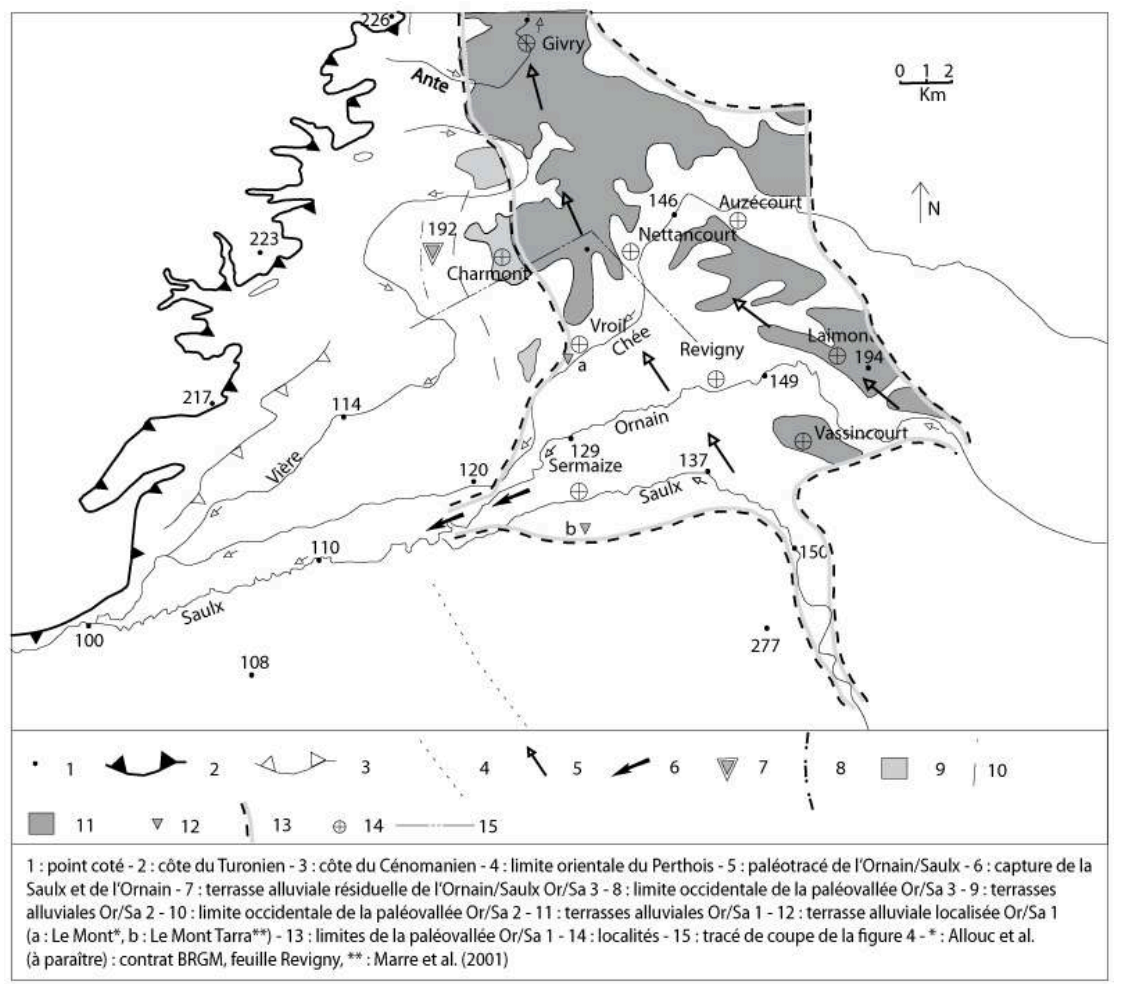

Figure 4 : Traces des alluvions anté-capture sur l'interfluve Chée-Ornain-Vière

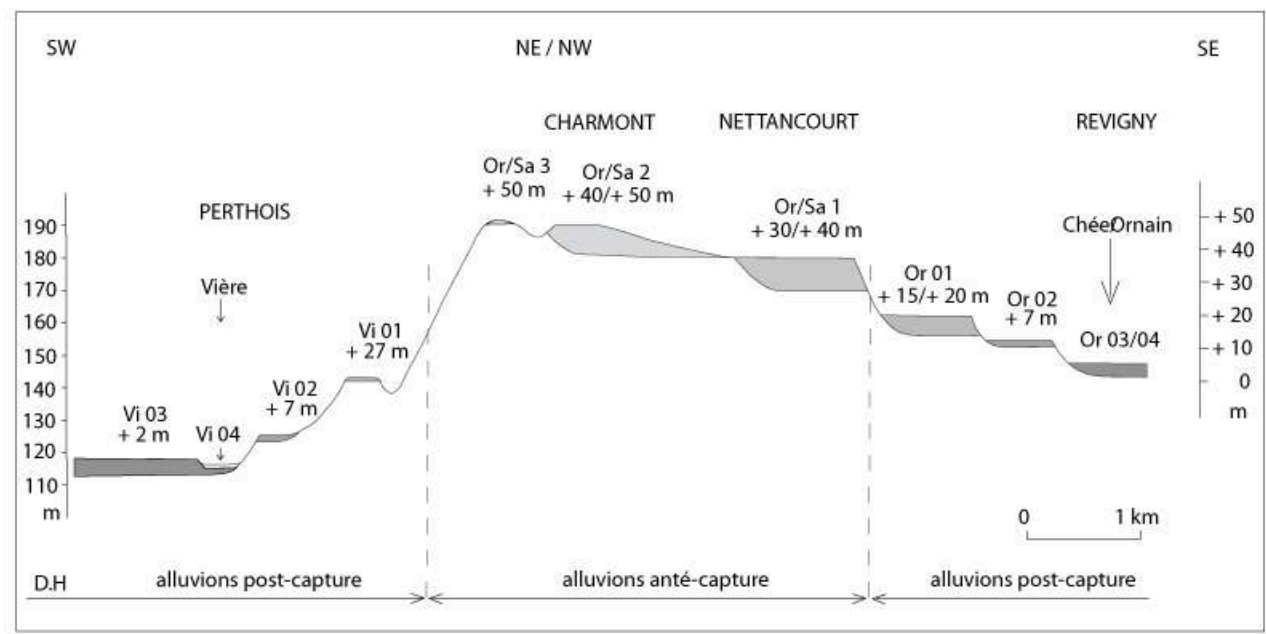

\section{Les alluvions anté-capture de l'Ornain/Saulx (Or/Sa 1)}

11 Deux coupes, situées à l'Ouest de Laimont respectivement au Nord et au Sud de la route départementale D75 ont permis d'observer in situ les alluvions F 1 (fig. 5, 6). 
Figure 5 : Variations longitudinales de faciès dans les alluvions anté-capture F1

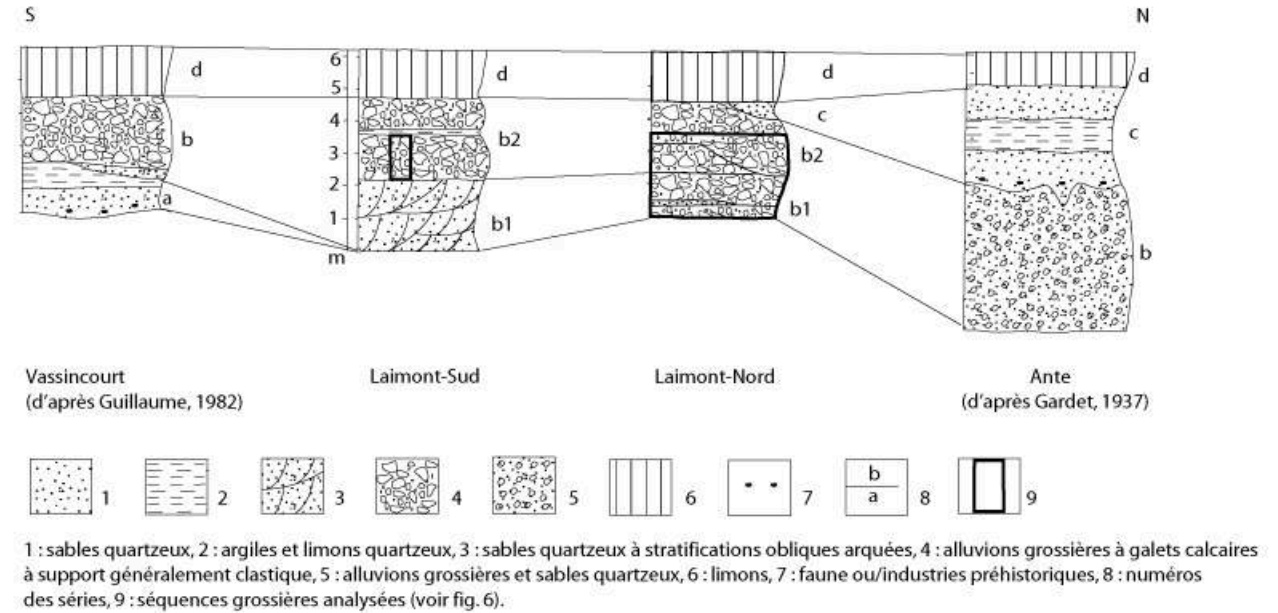

Figure 6 : Analyses granulométriques des alluvions grossières de la formation $\mathrm{F} 1$ de Laimont $6 \mathrm{~A}=$ séquences inférieures de Laimont-Nord, 6B = séquences inférieures de Laimont-Sud

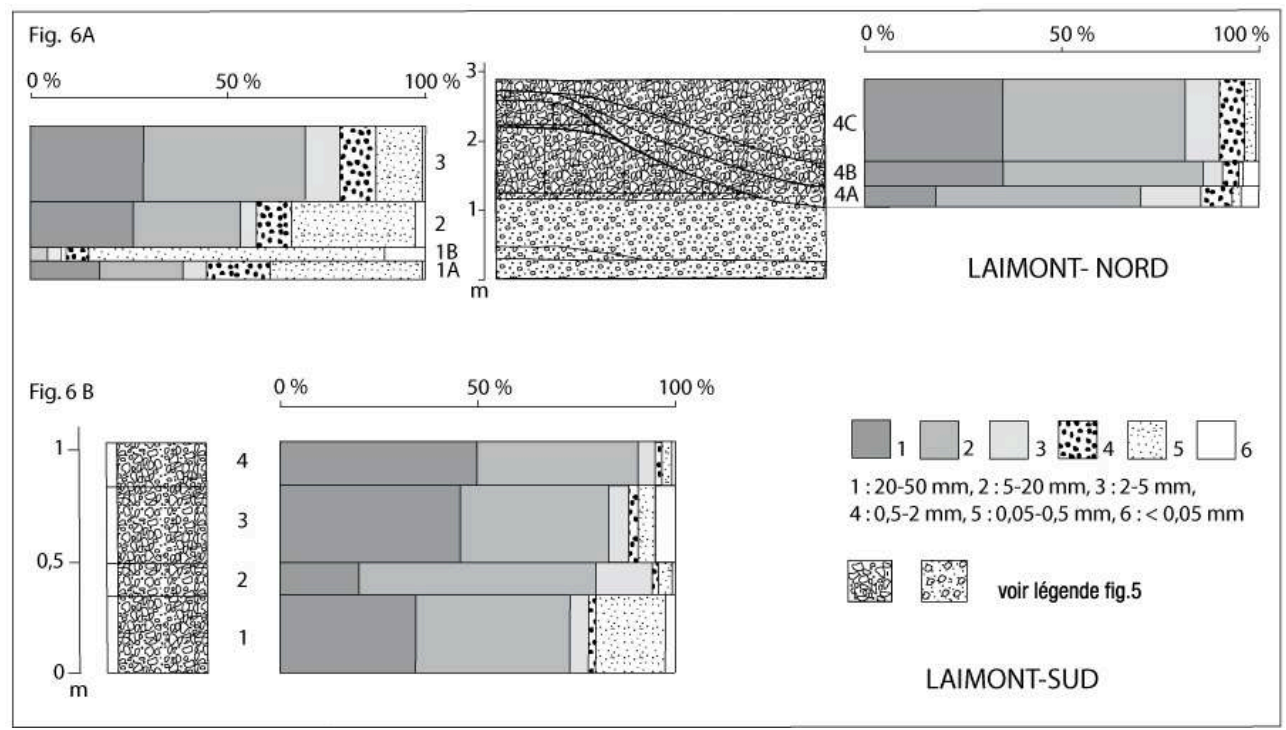

12 La première coupe (Laimont-Nord) est localisée dans la carrière de granulats des établissements Roussel. Elle montre, de bas en haut :

1. les argiles du Gault ;

2. des alluvions essentiellement grossières de $5 \mathrm{~m}$ d'épaisseur, constituées d'une succession de séquences de dépôts grossiers et sablo-graveleux d'épaisseur pluridécimétrique de couleur jaune (10 YR 8/6) à brun-jaune (10 YR 5/6 à 6/8) ou brun (10 YR 8/4). Ces dépôts sont organisés en strates horizontales (sous-série b1 : séquences $1,2,3)$ recoupées par de larges chenaux (sous-série b2 : séquence 4A, 4B, 4C) (fig. 6) ;

3. des alluvions grossières, à petites structures chenalisantes comblées de sables quartzeux (série c);

4. au sommet, 1,5 à $2 \mathrm{~m}$ de limons bruns décarbonatés, argileux à la base (série $\mathrm{d}$ ).

13 La seconde coupe (Laimont-Sud), visible jusqu'à la fin des années 1990, montrait 3 types de lithofaciès, où on pouvait observer de bas en haut des séquences alternativement grossières et sableuses à stratifications obliques arquées à pendage apparent vers le 
sud, des séquences grossières de $2 \mathrm{~m}$ d'épaisseur, et une couverture limoneuse non carbonatée.

Les analyses granulométriques effectuées sur les 2 coupes mettent en évidence la prépondérance des éléments grossiers. Ceux-ci constituent souvent plus des $3 / 4$ de l'échantillon (Laimont Sud : séquences 1 à 4 , Laimont-Nord : séquences 2 à $4 \mathrm{C}$ ), la part des galets de plus de $5 \mathrm{~mm}$ de diamètre représentant entre $50 \%$ et $90 \%$ du total pour ces séquences.

Les analyses montrent également un gradient granulométrique croissant, les valeurs de la médiane passant de $1,1 \mathrm{~mm}$ (séquence $1 \mathrm{~A}$ ) à $16 \mathrm{~mm}$ (séquence $4 \mathrm{C}$ ) dans la coupe de Laimont-Nord, et de $15 \mathrm{~mm}$ (séquence 1) à $20 \mathrm{~mm}$ (séquence 4) dans la coupe de Laimont-Sud.

En outre, dans la coupe de Laimont-Nord, l'analyse granulométrique réalisée à partir des 4 séquences de base montre que les alluvions grossières peuvent être subdivisées en 2 sous-séries. La sous-série basale $(\mathrm{b} 1)$ à séquences $(1 \mathrm{~A}, 1 \mathrm{~B}, 2)$, à matrice sableuse abondante contient moins de $60 \%$ d'éléments grossiers et la médiane est inférieure à $10 \mathrm{~mm}$. La sous-série sommitale (b2) montre des séquences contenant plus de $75 \%$ d'éléments grossiers et la médiane est proche ou supérieure à $10 \mathrm{~mm}$.

Cette observation indique que le dépôt des alluvions grossières a été effectué par des courants de plus en plus compétents, sous des paléo-environnements caractérisés par des contrastes saisonniers de plus en plus marqués.

A partir des 2 coupes de Laimont et des travaux antérieurs, on peut distinguer 4 séries dans les alluvions $\mathrm{F} 1$ et préciser leur évolution longitudinale (Gardet, 1937; Patte, 1937 ; Guillaume, 1982 ; fig. 5) :

1. la série fine basale (a) est représentée à Vassincourt, au Sud de l'Ornain, où elle est marquée par des apports uniquement locaux (issus du Crétacé inférieur, notamment Sables Verts albiens et Argiles du Gault);

2. la série grossière (b), constituée de galets de calcaire tithonien, est représentée dans toute la région;

3. les dépôts fins (c), apparaissant sous forme de chenaux à Laimont, forment, dans le bassin de l'Aisne, une série épaisse, sableuse et argileuse ;

4. les limons (d) sont omniprésents au-dessus des alluvions calcaires.

19 La distinction en 4 séries s'appuie sur quatre types d'arguments. Tout d'abord, la faune à Equus caballus et Bos primigenius et l'analyse palynologique réalisée dans la série fine (a) de Vassincourt indiquent l'existence d'un climat tempéré, celui-ci se détériorant progressivement (Guillaume, 1982). Ensuite, le paléoenvironnement périglaciaire de la série grossière (b) peut être confirmé par ses caractéristiques sédimentologiques: stratifications subhorizontales attestant des débits élevés, caractère grossier et mauvais classement des sédiments (Laimont-Sud : écart-type indice d'Inman - compris entre 0,94 et 1,50 phi), indices d'aplatissement des galets voisins de 3 (Laimont-Nord, séquence 4C : 2,966, Laimont-Sud, séquence $1: 3,071$ ). Dans le Paléo-Perthois, l'étalement des alluvions sur plusieurs kilomètres de largeur tend à montrer que le style fluvial était celui d'un réseau en tresses. En outre, les épaisseurs importantes d'alluvions grossières indiquent l'existence, dans le Barrois, d'une cryoclastie active et de longue durée couvrant une grande partie des périodes froides (Sommé, 1984). 
20 Les découvertes effectuées dans les alluvions : bifaces trouvés à Vassincourt, dans la série fine (a) (Guillaume, 1982), et les éléments de faune composite à Palaeloxodon antiquus et à Mammuthus primigenius du bassin de l'Aisne supérieure (séries b et c) (Gardet, 1937 ; Patte, 1937), datent les alluvions F1 du Pléistocène moyen. Plus précisément, les éléments de faune datent la série basale (a) de 330 à $300 \mathrm{ka}$ BP (Guillaume, 1982), plaçant celle-ci dans le stade isotopique 9 (Shackleton, 1987). Par conséquent, les alluvions grossières formant la série $b$ se rattachent au stade isotopique 8. Les séries fines $c$ et $d$, quant à elles, témoignent d'une moindre compétence des cours d'eau, et sans doute de contrastes saisonniers moins importants du régime hydrologique (Lécolle, 1984). Elles correspondent à des milieux tempérés continentaux, comme l'indiquent les travaux réalisés dans le bassin de la Somme (Antoine, 1990) ou celui de l'Yonne (Chaussé et al., 2000).

21 Il est donc probable que les 4 séries de la formation F1 correspondent à un alluvionnement de longue durée, commençant à la limite des stades isotopiques 9 et 8 et s'étendant pendant la plus grande partie du stade 8 .

\section{Profils longitudinaux et transversaux de l'Ornain/Saulx}

Les données de terrain et les forages carottés ont permis de reconstituer le profil longitudinal de l'Ornain/ Saulx de Gondrecourt-le-Château à Givry-en-Argonne (fig. 7). Le profil Or/Sa 1 était moins incliné que les profils des talwegs actuels : la valeur de la pente de la paléovallée Or/Sa 1 était d'environ 0,055 \% entre Laimont à Givry-enArgonne, celle du talweg actuel est de 0,23\% de Revigny-sur-Ornain à Heiltz-leMaurupt (fig. 1, 7).

Figure 7 : Les stades d'incision de la vallée de l'Ornain

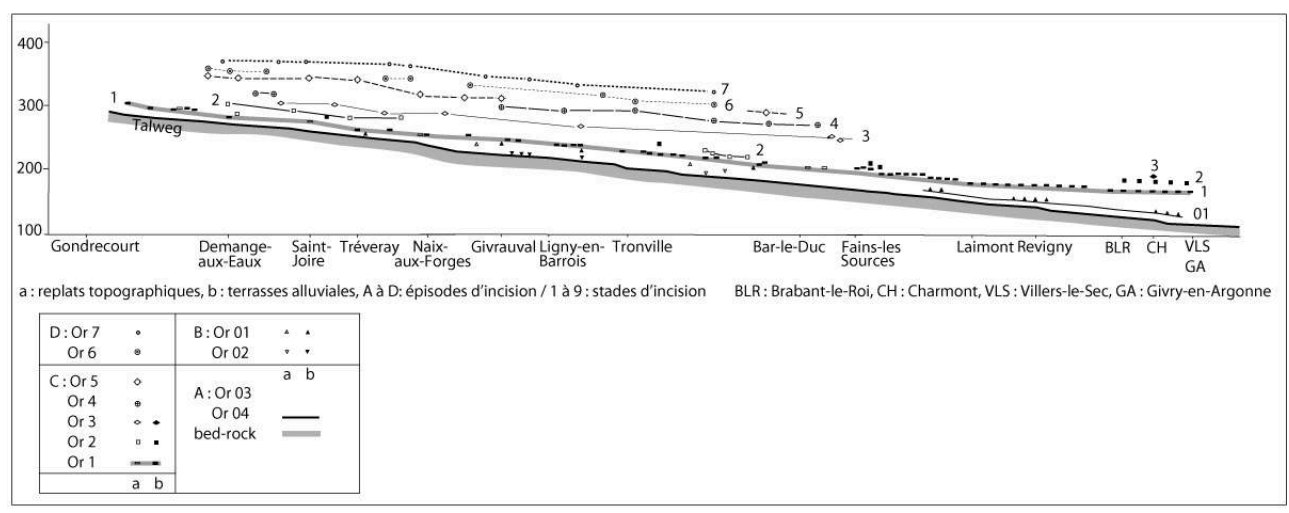

23 En outre, la paléovallée Or/Sa 1 était plus large que l'actuelle, atteignant $1 \mathrm{~km}$, au Sud de Bar-le-Duc (contre $500 \mathrm{~m}$ au maximum pour la vallée actuelle), et 15 à $16 \mathrm{~km}$ dans la région de Revigny. Au Nord de la localité, le prisme alluvial s'étendait vers le nord-nord-ouest, sur environ $25 \mathrm{~km}$ entre Revigny et Sainte-Menehould, où se situait la paléo-confluence entre l'Ornain/Saulx et l'Aisne, encaissée dans le massif de gaize de l'Argonne (Tricart, 1948).

24 Avant la capture, l'Ornain/Saulx coulait dans une large plaine alluviale, le PaléoPerthois, remblayée sur une grande épaisseur. Ce puissant remblaiement a eu pour effet d'exhausser le lit des deux cours d'eau dans la période précédant la capture (voir paragraphe III.4). 


\section{Les stades d'incision des vallées de l'Ornain, de la Saulx et de l'Ornain/Saulx}

\section{A. Stades d'incision et épisodes de creusement}

Dans le Barrois, en l'absence de formations alluviales, les nombreuses paléoformes d'érosion fluviatile (replats topographiques, rives concaves de méandres fossiles et vallons perchés) permettent de reconstituer les stades d'incision de l'Ornain et de la Saulx (fig. 8), en contrebas de la surface d'érosion sommitale reconstituée à partir des points hauts du relief actuel (Le Roux, 2000). Les stades d'incision sont regroupés en 4 épisodes.

Figure 8 : Les stades de creusement et l'incision dans les vallées de l'Ornain, de la Saulx et de I'Ornain/Saulx

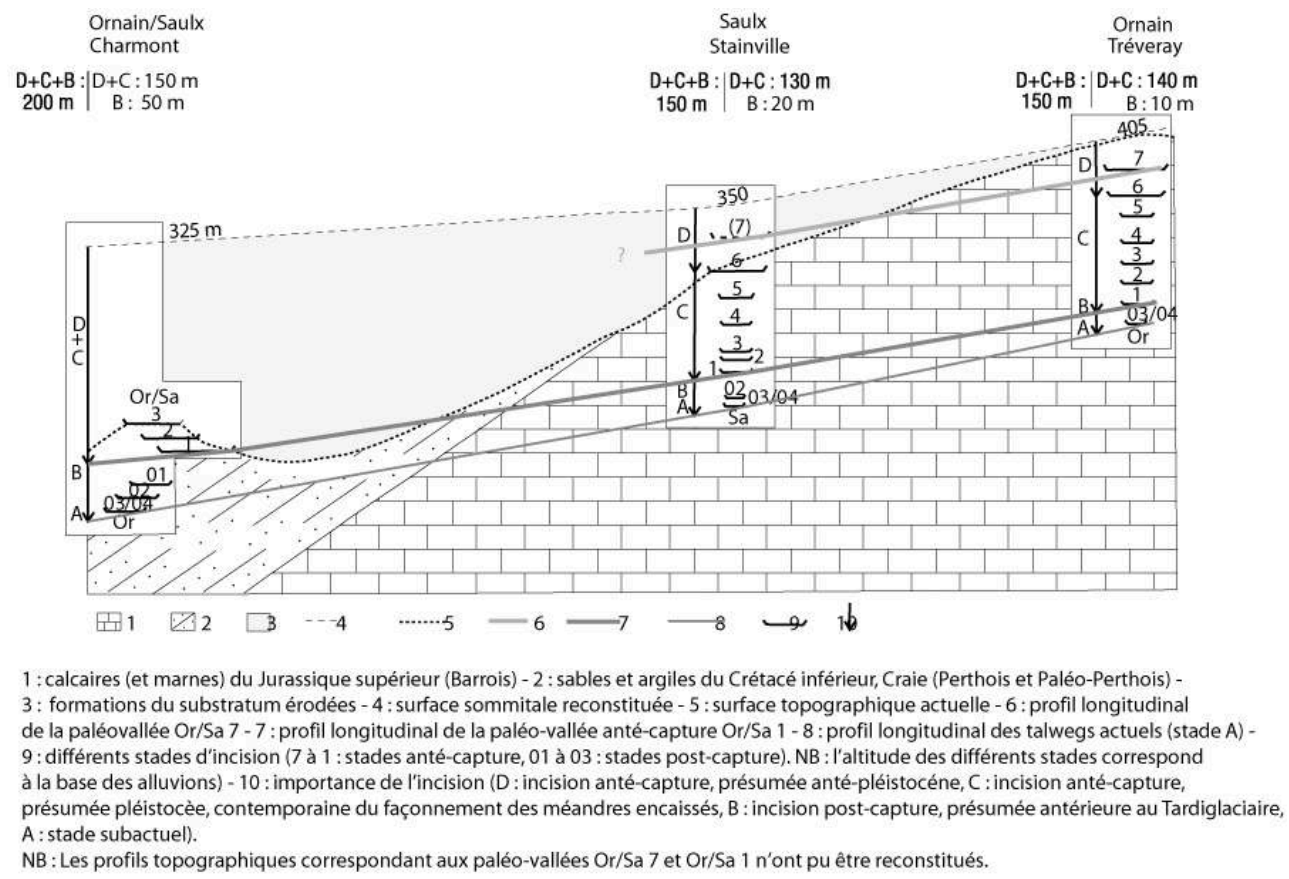

1. L'épisode très ancien (ou épisode D), uniquement représenté dans le Barrois, correspond à des paléovallées larges subrectilignes et peu encaissées dans le massif calcaire du Barrois. Les largeurs des paléovallées Or 7 et Or 6 étaient respectivement d'environ $4 \mathrm{~km}$ et $2 \mathrm{~km}$ dans le synclinal de Tréveray. Les deux paléovallées se situent actuellement à $20 \mathrm{~m}$ et à $40 \mathrm{~m}$ en contrebas de la surface sommitale. L'épisode très ancien coïncide ainsi avec un creusement modéré de l'Ornain et de la Saulx. A l'opposé, les 3 épisodes plus récents $(\mathrm{C}, \mathrm{B}, \mathrm{A})$ correspondent à des paléovallées à méandres encaissés, plus étroites, leur largeur étant inférieure à un kilomètre dans le synclinal de Tréveray.

2. Dans le Barrois, l'épisode ancien, anté-capture (ou épisode C) correspond à des paléovallées encaissées au tracé méandriforme (Or 5 et Or 4, Sa 5 et Sa 4), évoluant vers des paléovallées à méandres encaissés ( Or 3, Or 2, Or 1 et Sa 3 , Sa 2, et Sa 1), bien marquées à partir de Or 2 et Sa 2. Dans le Paléo-Perthois, les 3 dernières paléovallées anté-capture, jalonnées par des alluvions anciennes, se situent aujourd'hui en inversion de relief. Cet épisode correspond à l'essentiel du creusement, ce dernier s'élevant à $75 \mathrm{~m}$ dans la vallée de la Saulx et à $80 \mathrm{~m}$ dans la vallée de l'Ornain. 
3. L'épisode récent (ou épisode B), jalonné par 2 niveaux de terrasses (Or 01 et or 02, Sa 01 et Sa 02) correspond au creusement post-capture. Dans le Barrois, il coïncide avec des paléovallées à méandres encaissés plus étroites, tandis que dans le Perthois, elles correspondent à des terrasses étagées, situées à respectivement à +15/20 m et à +5/7 m.

4. L'épisode actuel (ou épisode $A$ ) correspond aux fonds de vallée actuels. Dans le Perthois, les lits mineurs des cours d'eau actuels (Or 04, Sa 04) sont incisés de 1 à $5 \mathrm{~m}$ dans le vaste remblaiement alluvial de fond de vallée (Or 03, Sa 03) (fig. 4).

\section{B. Les étapes du creusement des vallées}

Par analogie avec le bassin de la Seine moyenne et inférieure (Lautridou et al., 1999), de la Meuse lorraine (Pissart et al., 1997) ou de la Marne supérieure (Marre et al., 2000), les stades d'incision de l'épisode $D$ jalonnés par de larges gouttières peu encaissées, sont présumés d'âge fini-pliocène. En conséquence, la phase d'incision majeure $(C)$ se situerait au Pléistocène ancien et moyen. Les phases d'incision postérieures ( $B$ et $A$ ), se situent entre le Pléistocène moyen et le Weichsélien. En effet, le dernier grand épandage alluvial du Perthois peut être rapporté à la dernière période froide, comme dans les autres vallées de l'Est du bassin de Paris (Pastre et al., 1991; Lefèvre et al., 1993). L'incision de la nappe de fond de vallée, weichsélienne, semble générale dans tout le bassin de Paris. Par exemple, dans le bassin de la Marne inférieure, elle est incisée de $2 \mathrm{~m}$ entre le Tardiglaciaire et le Préboréal (Pastre et al., 1991). Plus précisément, deux phases d'incision ont été mises en évidence au Bölling et au début du Préboréal dans le bassin de la Seine (Pastre et al., 2000).

\section{Evaluation des taux d'incision}

On distinguera, d'une part, l'incision ante-capture, calculée à partir de l'altitude de la surface sommitale, d'autre part, l'incision post-capture, enfin, l'incision totale.

L'incision anté-capture présente des valeurs semblables dans les deux vallées de la Saulx et de l'Ornain : $130 \mathrm{~m}$ à Stainville, $140 \mathrm{~m}$ à Tréveray et $150 \mathrm{~m}$ dans la paléovallée de l'Ornain/Saulx. D'après l'âge présumé pliocène de la surface sommitale (Le Roux, 2000) et l'âge pléistocène moyen des alluvions F 1 (environ 250000 ans BP), on peut estimer le taux d'incision anté-capture à environ $3 \mathrm{~cm}$ ou $4 \mathrm{~cm} / 1000$ ans dans le Barrois et le Paléo-Perthois.

L'incision post-capture présente des valeurs plus élevées sur le site de capture (Perthois), que plus en amont (Barrois), les valeurs étant de $50 \mathrm{~m}$ à Sermaize-les-Bains, $30 \mathrm{~m}$ à Revigny-sur-Ornain, 20/25 m à Stainville, et $+10 / 15 \mathrm{~m}$ à Tréveray. Les taux d'incision post-capture s'élèvent ainsi respectivement à $20,12,8$ à 10 et 4 à $6 \mathrm{~cm} / 1000$ ans.

Toutefois, les valeurs plus élevées de l'incision post-capture sont dues également à la lithologie, puisque l'incision est plus importante dans les sables et argiles du Crétacé inférieur du Perthois, que dans les calcaires tithoniens du Barrois.

31 Si l'incision totale est nettement plus élevée dans le Perthois $(200 \mathrm{~m})$ que dans le Barrois (environ $150 \mathrm{~m}$ ), elle n'a pas eu les mêmes effets sur les paysages. Dans les calcaires tithoniens, elle s'est exercée verticalement, tandis que dans la craie et dans les formations argilo-marneuses du Néocomien, elle s'est accompagnée d'une ablation 
considérable des interfluves : $130 \mathrm{~m}$ de craie ont été érodés depuis le Pliocène dans le Paléo-Perthois.

\section{Modalités de la capture de l'Ornain-Saulx}

32 Ces conclusions montrent que la capture de l'Ornain-Saulx n'est que l'aboutissement d'un long processus d'érosion différentielle, mettant en relief les calcaires tithoniens et creusant les assises argilo-marneuses du Crétacé inférieur et la Craie (fig. 8). Cette érosion s'est effectuée surtout au Pléistocène, période qui a permis l'abaissement des interfluves par la gélivation de la Craie, comme en témoignent les « graveluches » - ou grèzes litées -, de la Champagne crayeuse (Laurain et al., 1995). Ce n'est donc que lorsque les interfluves situés à la limite des bassins versants de l'Ornain-Saulx et de la Marne ont été suffisamment bas topographiquement que la capture de la Saulx et de l'Ornain a pu se produire.

De toute évidence, cette capture est une capture complexe provoquée par de nombreux facteurs, comme dans le cas de la capture de la Haute Moselle (Harmand et al., 1995).

Ces facteurs peuvent être précisés en mettant en évidence le rôle joué par chacun des 4 segments de cours d'eau impliqués dans un processus de capture (Weisrock, 1997). La capture s'est effectuée en effet (fig. 9) :

- par contrôle aval du cours d'eau bénéficiaire, c'est-à-dire la Marne. Ainsi, dans le Perthois, la Marne a surcreusé sa vallée dans les formations argilo-sableuses du Crétacé inférieur, notamment dans les argiles du Gault dont l'épaisseur atteint $80 \mathrm{~m}$ (Stchépinsky, 1959) ;

36 - par érosion régressive du cours d'eau tributaire, la Vière, affluent de la Marne, alimenté par les aquifères de la côte de Champagne (Allouc et al., à paraître) et dont le bassin versant s'inscrit entièrement dans les formations marneuses de l'Albien et du Cénomanien faciles à déblayer ;

37 - par contrôle amont des 2 cours d'eau capturés, l'Ornain et la Saulx, dont le cours supérieur et moyen apparaît perché sur les calcaires tithoniens. La cryoclastie active dans le Barrois pendant les périodes froides du Quaternaire, notamment durant le stade isotopique 8, a généré une importante accumulation alluviale. Ces alluvions, déposées dans la dépression argilo-marneuse du Paléo-Perthois, ont ainsi provoqué un exhaussement du lit de la Saulx, de l'Ornain et de la Chée. Ajoutons le fait que des soutirages karstiques effectués de la Saulx vers la Marne (Jaillet, 2000) ont pu affaiblir les débits de la Saulx et ralentir son creusement avant la capture ;

38 - par contrôle aval du paléo-cours d'eau tronçonné, l'Aisne-Ornain-Saulx, dont la vallée était déjà encaissée dans la gaize dans la région de Sainte-Menehould au moment de la capture. Les difficultés de creusement du paléo-cours d'eau, surimposé dans la gaize de l'Argonne (Tricart, 1948) expliquent le caractère perché de l'Ornain-Saulx par rapport à la Marne et l'exhaussement important du paléo-cours d'eau incapable d'évacuer vers l'aval toutes ses alluvions. Il faut toutefois nuancer la résistance à l'érosion de la Gaize. Cette formation est seulement un peu plus résistante que les formations voisines. Mais, localement, elle peut être plus cimentée. 
Figure 9 : Les mécanismes de la capture de l'Ornain-Saulx

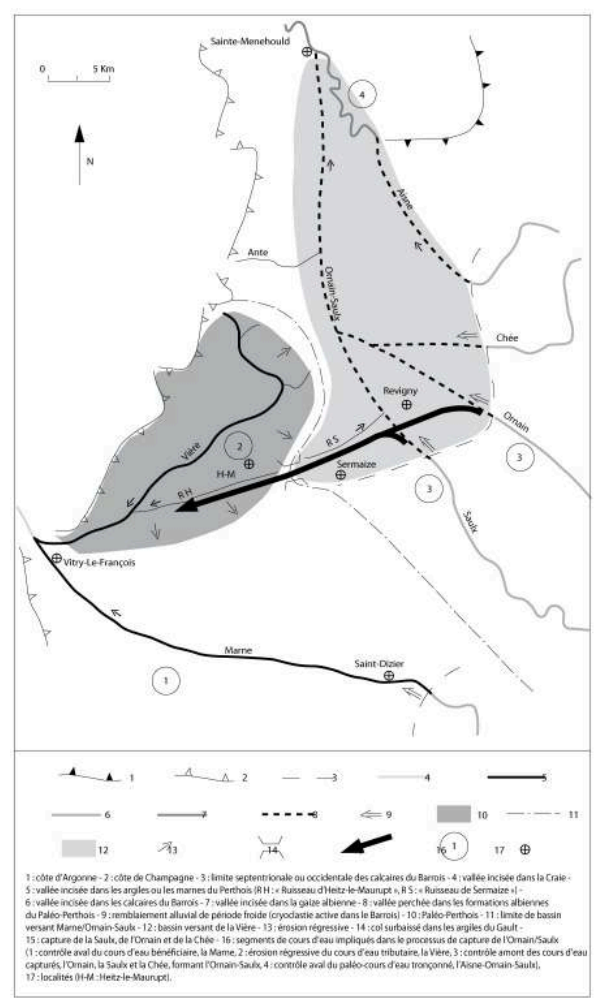

Il apparaît qu'à l'échelle du Quaternaire, les alluvions grossières de l'Ornain-Saulx ont eu un effet protecteur sur les paysages, comme en témoignent les larges placages alluviaux appartenant aux 3 dernières formations anté-capture, qui existent sur la feuille de Revigny-sur-Ornain (Allouc et al., à paraitre). A l'opposé, le creusement s'est effectué plus aisément dans le bassin de la Vière, dépourvu d'alluvions grossières.

La capture, préparée de longue date par l'érosion différentielle, favorisée par le rôle des facteurs climatiques et morphostructuraux, s'est réalisée dans la région de Sermaizeles-Bains, comme le montre la localisation des alluvions anté-capture situées au Sud de cette localité (Marre et al., 2001) et au Sud de Vroil (Allouc et al., à paraître). Leur localisation évoque la fossilisation d'une vallée affluente de la Saulx par la nappe antécapture F1. Les alluvions auraient ainsi ennoyé la limite de bassin versant surbaissée entre Ornain/Saulx et Marne, provoquant la capture par déversement de la Saulx et de l'Ornain dans le bassin de la Marne. Plus précisément, cette capture se serait effectuée le long de la vallée du "Ruisseau de Sermaize», affluent de la Saulx, dans celle du «Ruisseau de Heitz-le-Maurupt », affluent de la Vière (fig. 9).

\section{Conclusion}

Dans les vallées de l'Ornain et de la Saulx, une dizaine de stades de creusement ont été enregistrés pendant le Plio-Pléistocène, chaque stade étant le résultat d'une incision dans le substratum atteignant le plus souvent entre 10 et $20 \mathrm{~m}$, et d'une karstification des calcaires tithoniens (Jaillet, 2000). Le nombre de stades d'incision dont il subsiste une trace dans le paysage actuel est en fait peu élevé comparé à celui des autres grandes vallées de l'Est du bassin de Paris, comme celles de la Haute Moselle ou de la Meuse, dans lesquelles on compte plus de 20 stades d'incision (Pissart et al.,1997). Il est, 
par contre, plus élevé que pour la Marne (6 stades) sachant que l'organisation générale en 4 grands épisodes de creusement est la même (Lejeune et al., 2002).

Les valeurs moyennes de vitesses d'incision sur la période plio-pléistocène (comprises entre 3 et $12 \mathrm{~cm} / \mathrm{ka}$ ) concordent avec celles obtenues dans d'autres régions, comme celles des sections de vallées de la Seine moyenne $(6,5 \mathrm{~cm} / \mathrm{ka}$ depuis 300000 ans BP) et inférieure ( $5 \mathrm{~cm} / \mathrm{ka}$ depuis 800000 ans) (Lefebvre et al., 1994). Par contre la dynamique d'incision "effective " peut fortement varier au cours du temps pour une même section de vallée, en conséquence de réorganisation par capture, comme l'illustre le cas de la Saulx et de l'Ornain.

La capture de l'Ornain/Saulx est une capture complexe qui peut être datée de la fin d'un alluvionnement débutant dans l'intervalle isotopique 9 et se poursuivant dans le stade 8. La capture remonterait ainsi à environ 250000 ans BP. Cette capture apparaît donc nettement postérieure au détournement vers l'Oise et la Seine, de " l'Aisne-Bar " (Harmand et al., 1998), qui daterait d'environ 900000 ans (Pissart et al., 1997). La vallée de «l'Aisne-Ornain-Saulx » (Tricart, 1948) est également originale, puisqu'elle a subi une capture dans sa partie supérieure, et a au contraire détourné à son profit l'Aire, ancien affluent de la Meuse dans sa partie moyenne. L'évolution géomorphologique des marges du bassin de la Meuse devra cependant être précisée par la datation relative des deux captures de l'Ornain-Saulx et de l'Aire.

\section{BIBLIOGRAPHIE}

Allouc J., FAuvel P.-J., HaRmand D., Le RouX J. (à paraître). - Carte géologique à 1/50 000, feuille Revigny-sur-Ornain (190) et notice explicative. BRGM, Orléans.

ANTOINE P. (1990). - Chronostratigraphie et environnement du Paléolithique du bassin de la Somme. Publications du Centre d'Etudes et de Recherches préhistoriques. Université des Sciences et techniques de Lille Flandre-Artois, 2, $207 \mathrm{p}$.

BLACHE J. (1943). - Captures comparées. La vallée morte de la Bar et les cas voisins. Rev. Géo. Alpine, 1-37.

Blondeau A., Pomerol B., avec la collaboration de Bournieras M., Fauconnier D., Fauvel P.-J., MonCIARDINI C. (1988). - Carte géologique à $1 / 50$ 000, feuille de Sainte-Menehould (160) et notice explicative. BRGM, Orléans.

Bors P. (1903). - Sur les variations de la Meuse à l'époque quaternaire. Comptes-rendus Académie des Sciences CXXXVII : 85-89.

BRAVARD J.-P., PetiT F. (1997). - Les cours d'eau. Dynamique du système fluvial. Armand Colin, 222 p.

BUVIGNIER A. (1852). - Statistique géologique, minéralogique, métallurgique, et paléontologique du département de la Meuse, J.B. Baillère édit., Paris, 694 p. + atlas.

CAIlleux A., TRICART J. (1959). - Initiation à l'étude des sables et des galets, t. I, CDU, 376 p. 
Chaussé C., Limondin-Lozouet N., Occhietti S., Voinchet P., BACon J.-C. (2000). - La nappe alluviale de Soucy-les-Grandes-Pièces (Yonne, France) : reconstitution pluridisciplinaire du fonctionnement d'un cours d'eau du Pléistocène moyen. Géographie physique et Quaternaire, $54(2): 187-208$.

DAvis W.-M. (1895). - La Seine, la Meuse et la Moselle. Ann. de géographie, 25-49.

Denizot G. (1919). - Observations sur la Marne et l'Aisne. C.R. somm. Soc. géol. France, 133-134.

DESHAIES M., WEISROCK A. (1995). - Amplitude des méandres encaissées quaternaires et surface des bassins-versants dans le Nord-Est de la France : implications paléogéographiques. Geodinamica Acta, 8 (1) : 33-55.

DEMASSIEUX L. (1969). - Carte géologique à 1/50 000, feuille Vaubécourt (191) et notice explicative. BRGM, Paris.

GARDET G. (1937). - Les alluvions de l'Ornain en aval de Bar-le-Duc (feuille de Bar-le-Duc au 1/80 000). Bull. Soc. géol. Fr., 222-229.

Guillaume Ch. (1982). - Stations de surface du Paléolithique inférieur et moyen de Lorraine. Bull. de l'Assoc. Fr. pour l'Etude du Quaternaire, 2 et $3: 135-146$.

HARMAND D., FAUVEl P.-J. (2000). - Etat des connaissances des terrasses des paléo-vallées de «l'Aisne-Ornain-Saulx » et de « l'Aire-Bar ». In : Analyse de l'évolution morphologique des confins Meuse-Haute Marne. Acquisition des données de terrain pour quantifier la dynamique de l'érosion. Première phase de travaux. Rapport ANDRA D RP 0GES 00-001 (confidentiel) (commande ANDRA n 012962SGG du 16/11/99), pp. 56-90.

HARMAND D., FAUVEL P.-J. (2001). - L'incision du réseau hydrographique aux confins Meuse/Haute Marne : Etudes des stades de creusement des vallées de l'Ornain et de la Saulx. In : Rapport ANDRA DRP 0 G2R 01-001 (confidentiel). Volume des textes : 43-69, volume des figures : 62-115.

HARMAND D., PISSART A., KRooK L. (1998). - L'évolution du paléo-bassin de la Meuse : les enseignements des captures et leurs implications environnementales. In : Arbeiten aus dem Geographischen Institut der Universität des Saarlandes. Symposium « Problèmes de l'environnement en Saar-Lor-Lux », 157-173, 6 fig., 1 tab.

Harmand D., Weisrock A., Gamez P., Le Roux J., Occhietti S., Deshaies M., Bonnefont J.-C., Sary M. (1995). - Nouvelles données relatives à la capture de la Moselle. Revue Géographique de l'Est, T. XXXV, 3-4 (Actes du Colloque Paléoréseaux hydrographiques quaternaires, Nancy, 6-8 septembre 1995), 321-343.

JAILLET S. (2000). - Un karst couvert de bas-plateau : le Barrois. Structure-fonctionnementévolution. Thèse de Géographie physique. Université Michel de Montaigne (Bordeaux 3), 543 p.

LAURAin M., Guerin H., MARRE A., Richard J. (1995). - Processus génétiques à l'origine des formations de pente à graviers de craie en Champagne. Permafrost and Periglacial Processes, $6: 103-108$.

Laurain M., Menillet F., Pluchery E., avec la collaboration de Duermael G., Fauvel P.-J., Thevenin S. (1998). - Carte géologique à $1 / 50$ 000, feuille Monthois (134) et notice explicative. BRGM, Orléans, $56 \mathrm{p}$.

Lautridou J.-P., Auffret J.-P., Baltzer A., Clet M., Lecolle F., Lefebvre D., Lericolais G., Roblin-Jouve A., Balescu S., Carpentier G., Descombes J.-C., Occhietti S., Rousseau D.-D. (1999). - Le fleuve Seine, le fleuve Manche. Bull. Soc. géol. France, 170 (4) : 545-558. 
LECOLLE F. (1984). — Phases érosives et cycles sédimentaires : les alluvions de la Seine au Sud du Vexin. Quaternaire, $1.2 .3: 33-36$.

Lefebvre D., Antoine P., Auffret J.-P., LAutridou J.-P., Lecolle F. (1994). — Réponses de la Seine et de la Somme aux événements climatiques, eustatiques et tectoniques du Pléistocène moyen et récent : rythmes et taux d'érosion. Quaternaire, 5 (3-4) : 165-172.

LefeVre D., Heim J., Gillot E., Mouthon J. (1993). — Evolution des environnements sédimentaires et biologiques à l'Holocène dans la plaine alluviale de la Meuse (Ardennes, France). Premiers résultats. Quaternaire, 4 (1) : 17-30.

Lejeune O., Marre A., Devos A., Laurain M., Brulhet J. (2002). - Les phases d'incision de la vallée de la Marne entre Joinville et Vitry-le-François (Est du Bassin parisien, France). Revue Géographique de l'Est, en ce volume.

LE Roux J. (2000). - Structuration du Nord-Est du bassin de Paris. Bull. Inf. Géol. Bass. Paris, 37 (4) : 13-34.

Marre A., Laurain M., Devos A., Lejeune O., Sibileau l., Dolique F. (2001). - L'incision du réseau hydrographique aux confins Meuse/Haute Marne : étude des stades de creusement de la vallée de la Marne. Rapport ANDRA DRP 0 G2R 01-001 (confidentiel), Volume des textes : pp. 20-41, volume des figures : 8-61.

Marre A., Laurain M., Dolique F., Devos A. (2000). - Evolution géomorphologique de la vallée de la Marne, de Joinville à Sommeville. Rapport ANDRA D RP 0GES 00-001 (confidentiel) (commande ANDRA n 012962SGG du 16/11/99), pp. 91-115.

MAubeuge P.-L. (1965). - Carte géologique à 1/50 000, feuille Commercy (228) et notice explicative. BRGM, Paris.

MAUbeuge P.-L. (1970). - Carte géologique à 1/50 000, feuille Gondrecourt (266) et notice explicative. BRGM, Paris.

PÂQue A., CAILleuX A. (1946). - A propos des alluvions anciennes entre Ornain et Aisne. C.R. somm. Soc. géol. Fr., $9:$ 157-159.

Pastre J.-F., Cecchini M., Dietrich A., Fontugne M., Gauthier A., Kuzucuoglu C., Leroyer C., Limondin N. (1991). - L'évolution holocène des fonds de vallée au Nord-Est de la Région parisienne (France) : premiers résultats. Physio-Géo., 22-23 : 109-115.

Pastre J.-F., Leroyer C., Limondin-Lozouet N., Chausse C., Fontugne M., Gebhardt A., Hatte C., Krier V. (2000). - Le Tardiglaciaire des fonds de vallée du Bassin parisien (France). Quaternaire, 11 (2) : 107-122.

PATtE E. (1937). - Le Quaternaire dans la vallée de l'Aisne. Mém. Soc. géol. de France, n 32, 47 p. Pissart A., HARMAND D., KRook L. (1997). - L'évolution de la Meuse de Toul à Maastricht depuis le Miocène : corrélations chronologiques et traces des captures de la Meuse lorraine d'après les minéraux denses. Géographie Physique et Quaternaire, 51 (3) : 267-284, 10 fig., 1 tabl.

SHACKLETON N.J. (1987). - Oxygen Isotopes, Ice volume and Sea Level. Quarter. Sci. Rev., 6 : 183-190.

STCHÉPINSKY V. (1959). - Carte géologique à 1/50 000, feuille Saint-Dizier (226) et notice explicative. BRGM, Paris.

STCHÉPINSKY V. (1962). - Carte géologique à 1/50 000, feuille Bar-le-Duc (227) et notice explicative. BRGM, Paris. 
SommE J. (1984). - Signification dynamique et climatique des formations et terrasses fluviatiles dans l'Europe du Nord-Ouest. Bull. Ass. Fr. Et. Quat., 1, 2, 3 : 9-12.

TRICART J. (1948). - La partie orientale du Bassin de Paris. Etude morphologique. Thèse Paris (lettres) et SEDES éd. Paris, t. 1 : la genèse du bassin, pp. 1-210, t. 2 : l'évolution morphologique au Quaternaire, pp. 211-274.

WEISROCK A. (1997). - Cent ans après Davis : la question des captures et les réseaux hydrographiques quaternaires d'après les exemples de l'Europe du Nord-Ouest. Géographie Physique et Quaternaire, 51 (3) : 261-266.

\section{RÉSUMÉS}

Les travaux de cartographie montrent que les formations alluviales et les paléoformes fluviatiles des vallées de l'Ornain et de la Saulx permettent de reconstituer 11 stades de creusement pliopléistocène regroupés en 4 épisodes : $A$, très ancien ; $B$, ancien ; $C$, récent ; $D$, actuel. Deux d'entre eux $(\mathrm{A}, \mathrm{B})$ sont antérieurs à la capture de l'Ornain et de la Saulx par la Marne et les deux autres (C, D) lui sont postérieurs. Les alluvions anté-capture, bien conservées, ont été rattachées au Quaternaire moyen d'après la faune et l'industrie préhistorique. Les taux d'incision de la Saulx et de l'Ornain ont brusquement varié suite à leur capture par la Marne, événement survenu il y a environ $250 \mathrm{ka} \mathrm{BP}$. Estimées à environ 3 à $4 \mathrm{~cm} / \mathrm{ka}$ sur tout le secteur (dans le Paléo-Perthois comme dans le Barrois) pour la période anté-capture, les vitesses d'incision sont nettement plus élevées pour la période post-capture. Maximales $(12 \mathrm{à} 20 \mathrm{~cm} / \mathrm{ka})$ sur le site de capture, elles se réduisent progressivement d'aval en amont ( 8 à $10 \mathrm{~cm} / \mathrm{ka} \mathrm{à} \mathrm{Stainville} \mathrm{et} 4$ à $6 \mathrm{~cm} / \mathrm{ka}$ à Tréveray) et varient dans le détail en fonction des conditions liées à la nature du substratum, aux confluences et aux processus karstiques.

Mapping shows that the alluvial formations and fluvial palaeoforms of the Ornain and Saulx valleys can be used to reconstruct 11 stages of Plio-Pleistocene incision subdivided in to 4 episodes : A, very old ;B, old ; C, recent ; D, present. Two of them $(A, B)$ predate the capture of the Ornain and the Saulx by the Marne, and the two others (C, D) post-date it. The well-preserved pre-capture alluvial deposits were linked to the Mid-Quaternary by the fauna and prehistoric industry. The rates of incision of the Saulx and the Ornain varied abruptly following their capture by the Marne, an event which occurred about $250 \mathrm{ka} \mathrm{BP}$. Estimated at about 3 to $4 \mathrm{~cm} / \mathrm{ka}$ throughout the sector (in the Paleo-Perthois and the Barrois) for the pre-capture period, incision speed increased considerably during the post-capture period. Maximum rates (12 to $20 \mathrm{~cm} / \mathrm{ka})$ were recorded at the capture site and gradually decreased upstream ( 8 to $10 \mathrm{~cm} / \mathrm{ka}$ at Stainville and 4 to $6 \mathrm{~cm} / \mathrm{ka}$ at Tréveray), varying in detail according to conditions linked to the nature of the substrate, confluences and karstic processes.

Auf der Grundlage der in diesem Rahmen vorgenommenen Kartografier arbeiten lassen sich in den alluvialen Formationen und fluviatilen Paläo-Formen der Täler des Ornain und der Saulx 11 Einschnittstadien des Plio-Pleistozäns rekonstituieren, die sich wiederum in vier Abschnitte : A, sehr alt ; B, alt ; C, jung ; D, gegenwärtig, unterteilen. Zwei von ihnen (A, B) sind vor der Anzapfung des Ornain und der Saulx durch die Marne und die beiden anderen (C, D) danach anzusiedeln. Die gut konservierten Ablagerungen aus der Zeit vor der Anzapfung wurden entsprechend der Fauna und der prähistorischen Industrie dem mittleren Quartär zugeordnet. Die Einschnittwerte der Saulx und des Ornain haben sich infolge ihrer Anzapfung durch die Marne, der vor etwa $250 \mathrm{ka}$ BP erfolgte, erheblich geändert. Sie werden für die Periode vor der Anzapfung und für den gesamten Bereich (im Paläo-Perthois als auch im Barrois) auf 3-4 cm/ka 
geschätzt. In der Periode nach der Anzapfung sind die Einschnittswerte deutlich höher. Die Höchstwerte $(12-20 \mathrm{~cm} / \mathrm{ka})$ am Ort der Anzapfung nehmen Richtung flussaufwärts ab $(8-10 \mathrm{~cm} /$ ka in Stainville und 4-6 cm/ka in Tréveray) und variieren im einzelnen in Abhängigkeit von der Art des Untergrundes sowie der Zuflüsse und der Karsteinwirkungen.

\section{INDEX}

Mots-clés : Barrois, capture, Ornain, Paléo-Perthois, Saulx, stades d'incision

Keywords : Barrois, capture, Ornain, Palaeo-Perthois, Saulx, stages of incision

Schlüsselwörter : Anzapfung, Barrois, Einschnittstadien, Ornain, Paläo-Perthois, Saulx

\section{AUTEURS}

\section{DOMINIQUE HARMAND}

Laboratoire de Géographie, Campus Lettres-Sciences Humaines, Université de Nancy 2, BP 33-97,54015 Nancy Cedex, Dominique.Harmand@univ-nancy2.fr

\section{PIERRE-JEAN FAUVEL}

UMR 7566 G2R, Géologie et Gestion des Ressources Minérales et Energétiques, Université Henri Poincaré, (Nancy 1), BP 239, 54506 Vandœuvre-lès-Nancy

\section{STÉPHANE JAILLET}

UMR 7566 G2R, Géologie et Gestion des Ressources Minérales et Energétiques, Université Henri Poincaré, (Nancy 1), BP 239, 54506 Vandœuvre-lès-Nancy

\section{JACQUES LE ROUX}

UMR 7566 G2R, Géologie et Gestion des Ressources Minérales et Energétiques, Université Henri Poincaré, (Nancy 1), BP 239, 54506 Vandœuvre-lès-Nancy

JACQUES ALLOUC

Institut National Polytechnique de Lorraine, Ecole Nationale Supérieure de Géologie, BP 40, 54501 Vandœuvre-lès-Nancy

\section{JACQUES BRULHET}

ANDRA Direction Scientifique, 1/7, rue Jean-Monnet, 92298 Châtenay-Malabry

\section{MARCEL BROCANDEL}

ANDRA Direction Scientifique, 1/7, rue Jean-Monnet, 92298 Châtenay-Malabry 